\title{
Current anticoagulation therapy for sepsis-induced disseminated intravascular coagulation in Japan: results of a multicenter study using administrative data
}

\author{
Takeshi Umegaki, Miho Sekimoto, Hiroshi Ikai, Yuichi Imanaka ${ }^{\dagger}$
}

\begin{abstract}
Objective: Disseminated intravascular coagulation (DIC) is a serious complication associated with various underlying disorders, including sepsis. The aim of the current study was to investigate the status of therapy for patients with sepsis-induced DIC and to examine the association between 28-day mortality and use of anticoagulants. Methods: A multicenter cross-sectional study was performed from January 1, 2007 to December 31, 2008 in 45 ICUs in Japan. Using administrative data, 579 cases of sepsis-induced DIC were identified among patients who were admitted to an ICU, and these cases were used to assess the status of DIC therapy. The 28-day mortality was adjusted for the Critical care Outcome Prediction Equation (COPE) score, the Charlson comorbidity index and patient age, and associations with anticoagulants were then examined. Results: Protease inhibitors were used in 413 cases $(71.3 \%)$, and antithrombin, unfractionated heparin, and low molecular weight heparin/danaparoid were used in $313(54.1 \%), 385$ $(66.5 \%)$ and $201(34.7 \%)$ cases, respectively. The overall 28 -day mortality was $37 \%$. In a Cox proportional hazards regression model, the hazard ratio (HR) of unfractionated heparin was 1.41 , with a significant adverse effect on mortality $(P=0.02)$. In a similar analysis, the HRs for protease inhibitors, antithrombin and low molecular weight heparin/danaparoid were $0.86,0.90$ and 0.88 , respectively. These agents showed a tendency to reduce 28-day mortality, but the effect was not significant. Conclusions: A review of administrative data revealed that protease inhibitors were most frequently used in DIC anticoagulation therapy in ICUs in Japan. Unfractionated heparin was the only therapy to have a significant adverse effect on mortality.
\end{abstract}

Key words: (1) disseminated intravascular coagulation, (2) anticoagulants, (3) multicenter study

\section{Introduction}

Disseminated intravascular coagulation (DIC) is a complex, acquired life-threatening disorder characterized by massive systemic intravascular coagulation that leads to widespread deposition of fibrin in the circulation ${ }^{1)}$. Anticoagulation therapy has been suggested to be beneficial for DIC ${ }^{2}$, and a study on guidelines for this therapy was published in 2007 in Japan ${ }^{3)}$. The aims of the current retrospective study were to investigate the status of therapy for patients with sepsis-induced DIC in ICUs in Japan, and to evaluate the association between

This article is featured in "HIGHLIGHTS IN THIS ISSUE". Please see the issue of J Jpn Soc Intensive Care Med 2010; 17: 474-476. use of anticoagulants and 28-day mortality.

Methods
Cases and selection criteria
Data were obtained from the Quality Indicator/
Improvement Project (QIP), in which detailed adminis-
trative claim data were collected from institutions. In the
QIP, these data were analyzed to obtain numerical indices
for the healthcare process, patient outcomes, and
management efficiency to provide feedback to establish-
ments that participate voluntarily in the project. In the
current study, administrative data were surveyed from 45
acute care hospitals with an ICU. These data provided
information on the characteristics of patients and daily
medical care, thereby permitting collection of data for a
large population. The following selection criteria were

Department of Healthcare Economics and Quality Management, Kyoto University Graduate School of Medicine

Yoshida Konoe-cho, Sakyo-ku, Kyoto, Kyoto 606-8501, Japan

$\dagger$ Corresponding author
Submitted for publication May 22, 2009 Accepted for publication February 17, 2010 
Table 1 ICD-10 codes utilized for identification of septic cases

\begin{tabular}{ll}
\hline \hline Condition & Code \\
\hline Salmonella septicaemia & A02.1 \\
Septicaemic plague & A20.7 \\
Anthrax septicaemia & A22.7 \\
Erysipelothrix septicaemia & A26.7 \\
Listerial septicaemia & A32.7 \\
Streptococcal septicaemia & A40 \\
Other septicaemia & A41 \\
Actinomycotic septicaemia & A42.7 \\
Disseminated herpesviral disease & B00.7 \\
Candidal septicaemia & B37.7 \\
Disseminated coccidioidomycosis & B38.7 \\
Disseminated histoplasmosis capsulati & B39.3 \\
Disseminated blastomycosis & B40.7 \\
Disseminated paracoccidioidomycosis & B41.7 \\
Disseminated sporotrichosis & B42.7 \\
Disseminated aspergillosis & B44.7 \\
Disseminated cryptococcosis & B45.7 \\
Disseminated mucormycosis & B46.4 \\
Puerperal sepsis & O85 \\
\hline
\end{tabular}

ICD-10, International Classification of Diseases, 10th version.

used in the study: (1) primary diagnosis of sepsis (Table 1); (2) secondary diagnosis of DIC [code D65 in the International Classification of Diseases, 10th version (ICD-10)]; (3) age $\geq 20$ years old; and (4) entry into the ICU and hospital discharge from January 1, 2007 to December 31, 2008.

We excluded patients who were not treated with anticoagulation agents and those who died on the day on which anticoagulant therapy was initiated. Cases that required dialysis were also excluded, since anticoagulant agents such as protease inhibitors and unfractionated heparin are also administered in renal replacement therapy.

\section{Practice patterns in DIC anticoagulant therapy}

Anticoagulation therapy was defined as the use of a protease inhibitor (gabexate mesylate or nafamostat mesylate), antithrombin, unfractionated heparin, or low molecular weight heparin/danaparoid. Low molecular weight heparin and danaparoid sodium were included in one category because danaparoid sodium has stronger anti-Xa activity than unfractionated heparin. In this study, the therapeutic dose of unfractionated heparin for treatment of DIC was defined as at least 10,000 U/day. Use of a low dose of unfractionated heparin was excluded due to the difficulty of distinguishing usage of this dose between arterial monitoring and DIC therapy.

\section{Statistical analysis}

All statistical analyses were performed using SPSS software for Windows (Dr. SPSS-II, SPSS Japan Inc.). For calculation of the 28-day mortality, the onset of DIC was defined as the day on which anticoagulant therapy
Table 2 Hospital and patient backgrounds

\begin{tabular}{lc}
\hline \hline Hospital background & \\
Number of hospitals & 45 \\
Number of patients & 579 \\
Number of beds in hospital & $404.8 \pm 230.2$ \\
Patient background & $71.1 \pm 13.2$ \\
Age & 57.3 \\
Gender (male \%) & 86.2 \\
Hospital admission course & \\
(Emergency \%) & Frequency $(\%)$ \\
\hline Charlson comorbidity index & 45.6 \\
0 & 23.7 \\
1 & 19.3 \\
2 & 6.0 \\
3 & 2.6 \\
4 & 2.4 \\
5 & 0.3 \\
$\geq 6$ & $7.7 \pm 7.1$ \\
\hline Length of ICU stay (days) & $35.8 \pm 27.4$ \\
Length of hospital stay (days) & $22.8 \pm 17.8$ \\
Expected mortality (\%) & 37 \\
28-day mortality (\%) & \\
\hline
\end{tabular}

was started, since the true onset time could not be established from the administrative data. The association between anticoagulants and 28-day mortality was examined using a Cox proportional hazards regression model adjusted for the critical care outcome prediction equation (COPE) score ${ }^{4)}$, Charlson comorbidity index ${ }^{5}$ ) and patient age. A $P$ value below 0.05 was considered as significant. The COPE model is a risk-adjustment tool for use in ICUs that is constructed from 5 variables (age, unplanned admission, mechanical ventilation, hospital category, and admission diagnosis). This model provides the only prognostic severity score that can be obtained from administrative data alone, without use of physiological data. Previous work has shown that hospital mortality in critical care patients can be predicted using the COPE model [receiver operating characteristics area under the curve $($ ROC AUC $)=0.83-0.84]^{4}$ ). The Charlson comorbidity index, on which a higher score indicates greater severity, is a useful tool for measurement of the comorbid status or case mix in health care databases and has been adapted for use with ICD-10 data ${ }^{6}$.

\section{Results}

\section{Background}

Data were examined for 37,456 patients who were treated in 45 ICUs. Among these patients, 724 (1.9\%) cases of sepsis-induced DIC were identified, but 145 (20\%) fulfilled the exclusion criteria, leaving 579 (80\%) that were included in our analysis. The patient and hospital backgrounds are shown in Table 2. The mean 
Anticoagulation therapy in DIC

Table 3 Use of anticoagulant agents in cases of DIC

\begin{tabular}{lc}
\hline \hline Anticoagulant agent (alone or in combination) & Number of cases (\%) \\
\hline Protease inhibitor & $413(71.3)$ \\
Antithrombin & $313(54.1)$ \\
Unfractionated heparin & $385(66.5)$ \\
Low molecular weight heparin/danaparoid & $201(34.7)$ \\
\hline
\end{tabular}

$\mathrm{n}=579$ (45 ICUs).

Table 4 Relationship between anticoagulant agents and adjusted 28-day mortality (Cox proportional hazards regression model)

\begin{tabular}{lccc}
\hline \hline Anticoagulant agent & HR & $95 \%$ CI & $P$ value \\
\hline Unfractionated heparin & 1.41 & $1.06-1.87$ & $0.02 *$ \\
Protease inhibitor & 0.86 & $0.63-1.16$ & 0.32 \\
Antithrombin & 0.90 & $0.67-1.20$ & 0.46 \\
Low molecular weight heparin/danaparoid & 0.88 & $0.59-1.31$ & 0.53 \\
\hline
\end{tabular}

$*: P<0.05$.

CI, confidence interval; HR, hazard ratio.

age of the patients was approximately 71 years old and the expected mortality in the COPE model was $22.8 \%$. More than half of the patients had a Charlson comorbidity index of 0 or 1 . The mean length of ICU stay was 7.7 days and the mean length of hospital stay was 35.8 days.

\section{DIC anticoagulation therapy}

The frequencies of use of anticoagulation therapies are displayed in Table 3. A protease inhibitor was used in 413 of the 579 cases $(71.3 \%)$, and antithrombin, unfractionated heparin, and low molecular weight heparin/ danaparoid were used in $313(54.1 \%), 385$ (66.5\%) and $201(34.7 \%)$ cases, respectively. These interventions were combined in various ways in many cases.

\section{Cox proportional hazards regression model}

The 28-day mortality was $37 \%$. After adjustment for the COPE score, Charlson comorbidity index and patient age, unfractionated heparin had a significant adverse effect on the 28-day mortality [hazard ratio $(\mathrm{HR})=1.41$, $P=0.02]$. In contrast, protease inhibitors, antithrombin and low molecular weight heparin/danaparoid showed a tendency to reduce the 28-day mortality, but this effect was not significant (Table 4).

\section{Discussion}

Wada ${ }^{3)}$ suggested guidelines for DIC treatment in 2007 to facilitate evidence-based practice of anticoagulation therapy. In these guidelines, antithrombin was recommended over protease inhibitors and low molecular weight heparin/danaparoid, but our results suggest that protease inhibitors are used more frequently than antithrombin in current practice. We also found that low molecular weight heparin/danaparoid is used less commonly than other anticoagulant agents. Thus, there is a discrepancy between the guidelines and current practice, which may be due to the historical development of DIC anticoagulant agents in Japan and the absence of a high level of evidence in support of anticoagulant therapy for DIC ${ }^{7)}$. Protease inhibitors were developed in the 1980s in Japan and unfractionated heparin has traditionally been used as an anticoagulant. In contrast, use of low molecular weight heparin/danaparoid has been relatively uncommon.

Evidence for use of unfractionated heparin for DIC has not been obtained, but this agent has traditionally been administered as a conventional indication ${ }^{3}$. We found that administration of unfractionated heparin is less frequent than that of protease inhibitors, and more frequent than that of antithrombin in Japan. Furthermore, unfractionated heparin had a significant adverse effect on mortality, consistent with the lower level of evidence in the guidelines. This unfavorable result with unfractionated heparin may be caused by complications of hemorrhage or use in cases with severe bleeding. However, the presence of thrombosis often requires use of unfractionated heparin based on risks and benefits, and therefore use of this agent is likely to continue in DIC cases. Previous trials of anticoagulants for DIC have often used unfractionated heparin as a control ${ }^{8), 9)}$. An appropriate control group for a clinical study should be based on standard or usual practice, but this concept is difficult to define ${ }^{10)}$. Our results suggest that future trials should consider using protease inhibitors or antithrombin as the control, since most physicians in multiple centers in Japan prefer these therapies for DIC. These findings are also consistent with the guidelines.

There is little evidence that shows that anticoagulation agents significantly reduce mortality ${ }^{2), 11) \sim 14)}$. Our analysis revealed a small trend for reduction of the 28-day mortality with use of these agents, but the effect 
was not significant, as also found in previous studies. A large-scale trial in cases with severe sepsis supported a slight, but not significant, benefit of low-dose heparin for reducing 28-day mortality, but underscored the importance of heparin withdrawal in cases that involved DIC and abnormal coagulation ${ }^{14)}$. In this study, we were unable to assess the effect of low-dose heparin since this treatment was excluded from our analysis.

The difference between the expected mortality and the 28-day mortality may have been due to the longer period of 28 days used in our study, in contrast to the period of hospitalization used in other studies. Expected mortality in the COPE model obtained from ICU data in Australia and New Zealand was defined as hospital mortality, but acute care hospitals in Japan traditionally include acute care, sub-acute care, and nursing-home care. Therefore, the length of the hospital stay in Japan is generally reported to be longer than that in other developed nations ${ }^{15)}$. However, the length of hospital stay for patients with septic DIC may be less than 28 days in other countries, and this might account for the difference between the expected and actual 28-day mortality in our study.

There are several limitations in the present study. First, most importantly, the administrative data did not include physiological data, the International Society on Thrombosis and Haemostasis (ISTH) DIC score, the Japanese Association for Acute Medicine (JAAM) DIC score, the DIC score developed by the Ad Hoc Group of the Japanese Ministry of Health and Welfare (JMHW), or a severity score for intensive care based on APACHE II or SAPS II. These scores reflect the severity of DIC, are used to predict patient outcomes ${ }^{16) \sim 18)}$, and can lead to early diagnosis and improved treatment when applied appropriately in clinical management of DIC ${ }^{19)}$. This lack of information on DIC scores is the main disadvantage of using administrative data. However, evaluation of the severity of critical care patients using the COPE model eliminated this concern, and we also adjusted for comorbidity using the Charlson comorbidity index. Second, while we had detailed data for anticoagulant use, we were unable to assess the protocol in each ICU. Therefore, we were unable to evaluate the association between mortality and each practice pattern, despite the large apparent variation in practice. Finally, the study was not performed as a randomized controlled study or a prospective study, suggesting that the results might provide a lower level of evidence. However, it was offset by the advantage of data collection from a large population with reduced effort and time.

\section{Conclusion}

In summary, current treatment for sepsis-induced DIC in Japan commonly includes use of protease inhibitors. A tendency for improvement of outcome was found with use of protease inhibitors, antithrombin, and low molecular weight heparin/danaparoid, whereas unfractionated heparin had a significant adverse effect on 28-day mortality.
The work in the manuscript was presented at the 36th Annual Meeting of the Japanese Society of Intensive Care Medicine in Osaka, 2009.

\section{Acknowledgements}

The authors are grateful for the voluntary participation of the hospitals in the Quality Indicator/Improvement Project (QIP). The hospitals also participated in or cooperated with the Diagnosis Procedure Combination (DPC) system in Japan. The datasets in QIP are managed under the informational security system of ISO27001. The Institutional Review Board of the Faculty of Medicine at the Graduate School of Medicine of Kyoto University approved QIP and this study.

\section{Conflict of Interest and Declarations}

All authors declare no conflicts of interest. The design, data collection and analysis, and writing of the manuscript were performed by all four authors. The corresponding author takes full responsibility for the validity of the data.

\section{References}

1) Kruger D. Acute systemic disseminated intravascular coagulation: managing a complex medical condition. JAAPA 2006;19:28-32.

2) Wiedermann CJ. Clinical review: molecular mechanisms underlying the role of antithrombin in sepsis. Crit Care 2006;10:209-17.

3) Wada H. Study on guidelines for the treatment of DIC. Rinsho Ketsueki 2007;48:377-85.

4) Duke GJ, Santamaria J, Shann F, et al. Critical care outcome prediction equation (COPE) for adult intensive care. Crit Care Resusc 2008;10:41.

5) Charlson ME, Pompei P, Ales KL, et al. A new method of classifying prognostic comorbidity in longitudinal studies: development and validation. J Chronic Dis 1987;40:373-83.

6) Sundararajan V, Henderson T, Perry C, et al. New ICD-10 version of the Charlson comorbidity index predicted in-hospital mortality. J Clin Epidemiol 2004;57:1288-94.

7) Wada H, Asakura H, Okamoto K, et al; Japanese Society of Thrombosis Hemostasis/DIC subcommittee. Expert consensus for the treatment of disseminated intravascular coagulation in Japan. Thromb Res 2010;125:6-11.

8) Aramoto H, Saito H, Shigematsu H, et al. Synthetic protease inhibitors in the treatment of disseminated intravascular coagulation. Nippon Rinsho 1993;51:93-8.

9) Sakuragawa N, Hasegawa H, Maki M, et al. Clinical evaluation of low-molecular-weight heparin (FR-860) on disseminated intravascular coagulation (DIC)--a multicenter co-operative double-blind trial in comparison with heparin. Thromb Res 1993;72:475-500

10) Brower RG, Matthay M, Schoenfeld D. Meta-analysis of acute lung injury and acute respiratory distress syndrome trials. Am J Respir Crit Care Med 2002;166:1515-7.

11) Sakuragawa N, Hasegawa $H$, Maki M, et al. Clinical evaluation of low molecular weight heparin (FR-860) on disseminated intravascular coagulation (DIC) - a multicenter cooperative double-blind trial in comparison with heparin-. Rinsho Iyaku 1992;8:423-53.

12) Warren BL, Eid A, Singer P, et al; KyberSept Trial Study Group. Caring for the critically ill patient. High-dose antithrombin III in severe sepsis: a randomized controlled trial. JAMA 2001;286:1869-78. 
13) Nishiyama T, Matsukawa T, Hanaoka K. Is protease inhibitor a choice for the treatment of pre- or mild disseminated intravascular coagulation? Crit Care Med 2000;28:1419-22.

14) Levi M. Disseminated intravascular coagulation. Crit Care Med 2007;35:2191-5.

15) Ikegami N, Campbell JC. Medical care in Japan. N Engl J Med 1995;333:1295-9.

16) Gando S, Saitoh D, Ogura H, et al. Natural history of disseminated intravascular coagulation diagnosed based on the newly established diagnostic criteria for critically ill cases: Results of a multicenter, prospective survey. Crit Care Med 2008;36: 145-50.

17) Bakhtiari K, Meijers JCM, de Jonge E, et al. Prospective validation of the International Society of Thrombosis and Haemostasis scoring system for disseminated intravascular coagulation. Crit Care Med 2004;32:2416-21.

18) Angstwurm MW, Dempfle CE, Spannagl M. New disseminated intravascular coagulation score: A useful tool to predict mortality in comparison with Acute Physiology and Chronic Health Evaluation II and Logistic Organ Dysfunction scores. Crit Care Med 2006;34:314-20.

19) Gando S, Iba T, Eguchi Y, et al. A multicenter, prospective validation of disseminated intravascular coagulation diagnostic criteria for critically ill cases: Comparing current criteria. Crit Care Med 2006;34:625-31. 